whose perfectly normal menstrual history practically excludes anorexia nervosa. The same diagnostic reasoning, obviously, cannot be used in thin subjects who have primary amenorrhoea of whatever cause, including $\mathrm{XY}$ gonadal dysgenesis.

Secondly, as 'body image distortion' is nonspecific, poorly defined and found in a significant proportion of normal Western females too (Hsu \& Sobkiewicz, 1991), the significance that can be attached to it, in the absence of other convincing anorexic features, is meagre as in the case reported. Besides, other anorexic behaviours such as bingeeating, self-induced vomiting or laxative abuse were absent in the patient.

In so far as the exact aetiology of anorexia nervosa remains unclear and its diagnosis thus depends on the constancy of association of a particular constellation of clinical features, the above three factors may make the diagnosis of anorexia nervosa an enigmatic one in subjects with XY gonadal dysgenesis and psychopathology. Therefore, while agreeing with the authors that anorexia nervosa, in the synergistic presence of other psychopathology, may stem from the biological and psychological confusion caused by XY gonadal dysgenesis, I would also like to add a further source of confusion to this potential association, namely, diagnostic confusion. Prudence, especially among those of us inclined to unearth anorexia nervosa, is suggested.

Hsu, L. K. G. \& SOBKIEwICZ, T. A. (1991) Body image disturbance: time to abandon the concept for eating disorders? International Journal of Eating Disorders, 10, 15-30.

World Health Organization (1987) ICD-10: 1986 Draft of Chapter V-Categories FOO-F99. Mental, Behavioural and Developmental Disorders. Geneva: World Health Organization, Division of Mental Health.

Department of Psychiatry

SING LEE

Chinese University of Hong Kong

Shatin

Hong Kong

\section{Methylfolate and psychiatric illness}

SIR: We would like to echo the comments of Anderson et al (Journal, January 1992, 160, 130) on the interesting paper by Godfrey et al (1990) concerning the enhancement of recovery from psychiatric illness by methylfolate.

Apart from the lack of sufficient information regarding changes on the Hamilton Rating Scale for Depression, it should be noted that the use of this scale and the Beck Depression Inventory as a measure of changes in schizophrenic patients $(41 \%$ of the total sample) was inappropriate. The lack of correlation was therefore expected. We wondered why more suitable and even simpler scales for this group of patients, such as the Brief Psychiatric Rating Scale, were not used. Instead, only a crude four-point clinical outcome scale was employed. It is quite difficult, for example, to differentiate between a score of '2' (residual symptoms/socially recovered) and ' 3 ' (pronounced impairment without full social recovery). Furthermore, the baseline ratings of the two groups at month zero were not reported and the question of their comparability remained. Thus, the difference between the methylfolate and placebo groups at one month, as measured by the clinical outcome scale, may partly be due to selection bias, or the initial clinical effect of methylfolate. Finally, the authors did not clearly state whether there was any change in dosage and regime of medication or the use of non-pharmacological therapy throughout the trial. This is noteworthy because the clinical outcome scores of the placebo group, for unclear reasons, showed no overall improvement after six months of psychiatric treatment. Instead, both depressed and schizophrenic patients on placebo remained markedly impaired (bordering on score ' 3 ').

Despite their potential importance, folate studies among non-Western populations are virtually nonexistent. Recently, we measured the folate level of 46 Chinese out-patients on chronic lithium treatment, and found that practically none of them had low serum or erythrocyte folate (Lee et al, 1992). Yet, within the normal range of serum folate $(7-39 \mathrm{nmol} /$ $1)$, patients $(n=14)$ with a higher serum folate level $(33.4 \mathrm{nmol} / \mathrm{l})$ were found to have a lower one-year affective morbidity than those $(n=32)$ with a lower folate $(26.0 \mathrm{nmol} / \mathrm{l})$. This is the first Chinese study to strengthen the suggestion that it may be a high rather than normal folate level which is associated with lower psychiatric morbidity (Coppen et al, 1986). In the study of Godfrey et al, the significant difference in outcome between the folate and placebo groups at six months, despite the already normalised red cell folate level of the latter, gives further support to this possibility.

The folate debate will continue but appears a promising one. The current research findings, as Procter (1991) put it, "should surely warrant the attention of all practising psychiatrists and if confirmed may deserve wider general application". A controlled therapeutic trial, for example, is valuable for finding out whether Chinese patients with normal folate status may also benefit from folate pharmacotherapy. If it works, it may not really matter much, as in the case of psychotropics generally, to find out why it does so. 
Coppen, A., Chaudhry, S. \& Swade, C. (1986) Folic acid enhances lithium prophylaxis. Journal of Affective Disorders, 10, 9-13.

Godfrey, P. S. A., TOONE, B. K., CARNeY, M. W. P., et al (1990) Enhancement of recovery from psychiatricillness by methylfolate. Lancet, 336, 392-395.

LeE, S., Chow, C. C., Shek, C. C., et al (1992) Folate concentration in Chinese psychiatric outpatients on longterm lithium treatment. Journal of Affective Disorders (in press).

Procter, A. (1991) Enhancement of recovery from psychiatric illness by methylfolate. British Journal of Psychiatry, 159, 271-272.

YUN KWOK WING

SING LEE

Prince of Wales Hospital

Chinese University of Hong Kong

Hong Kong

\section{Christmas disease and major affective disorder}

SIR: We were interested to read Gill et al's description of a pedigree cosegregating Christmas disease and major affective disorder (Journal, January 1992, 160, 112-114). We would like to report a further family in which both disorders are segregating.

The proband, a 44-year-old man, has laboratoryproven Christmas disease and DSM-III-R bipolar disorder. His 40-year-old sister is a carrier of the Christmas disease gene and has DSM-III-R lifetime diagnoses of major depression and alcohol abuse. Her eldest son, who is 19 years old, has laboratoryproven Christmas disease and DSM-III-R major depression and alcohol abuse. The proband's father, who is 67 years old, is haematologically normal but had a single episode of DSM-III-R major depression at the age of 47 years. The proband's mother, who is 68 years old, is a carrier of the Christmas disease gene and had a two-year episode of depression at the age of 30 years which necessitated psychiatric referral but which only satisfies DSM-III-R criteria for depression, not otherwise specified. The proband has a haematologically normal son and four daughters who are obligate carriers of the Christmas gene. The proband's sister has two other sons in addition to the one already described: one who has Christmas disease and the other who is haematologically normal. None of these seven children has expressed affective disorder but all are below the age of 18 years and, thus, they are only starting to approach the risk period.

This pedigree is consistent with genetic linkage between the Christmas disease gene and a susceptibility locus for affective disorder but does not offer strong support for this hypothesis because of: (a) the small size of the pedigree, and (b) complications in interpretation introduced by assortative mating between the proband's parents. Like Dr Gill et al we examined the proband's karotype and performed molecular genetic studies on the proband's DNA in the region of the factor IX gene and found no evidence of any abnormality. Thus, it is unlikely that a single mutation such as a large deletion caused both disorders. Clearly our small pedigree may have arisen by chance but like several other pedigrees in the literature it focuses interest on the possibility of an affective disorder susceptibility locus in the $\mathrm{Xq} 27-28$ region.

Assuming no population association between Christmas disease and bipolar disorder, we estimate that there should be at least five men with both disorders in the United Kingdom. Drs Gill et al have reported one and we have reported another. Perhaps investigation of the families of some of the other doubly affected probands may further inform the continuing debate about the existence of $\mathrm{X}$-linked susceptibility loci in affective disorder (Hebebrand, 1992).

Hebebrand, J. (1992) A critical appraisal of X-linked bipolar illness. Evidence for the assumed mode of inheritance is lacking. British Journal of Psychiatry, 160, 7-11.

Nick Craddock

University of Birmingham

Queen Elizabeth Psychiatric Hospital

Birmingham B15 2QZ

Department of Psychological Medicine and

MiKe OWEN

Institute of Medical Genetics

University of Wales College of Medicine

Heath Park

Cardiff CF4 $4 X N$

\section{Survivors of disaster}

SIR: Dr Plummer's critique (Journal, March 1992, $160,420-421$ ) of our study of causal attributions and psychiatric symptoms in survivors of the Herald of Free Enterprise disaster (Journal, October 1991, 159, 542-546) is based on a misunderstanding of our work and on misapplications of statistical principles.

He begins, for example, by listing a number of "obvious methodological problems". Our sample, which we described carefully and in some detail, was certainly small and unrepresentative, but it is not clear how this fact alone could account for the pattern of findings. He then reproaches us for using correlation rather than regression analysis, omitting to mention that we used partial correlation analysis (regression is based on partial correlations). This confusion between correlations and partial correlations may also account for his puzzling charge that we had 